\title{
Detection of hTERC and c-MYC genes in cervical epithelial exfoliated cells for cervical cancer screening
}

\author{
TIAN LI, LIANGDAN TANG, DUHONG BIAN, YING JIA, XIN HUANG and XINHUA ZHANG \\ Department of Obstetrics and Gynecology, The First Affiliated Hospital of Chongqing Medical University, \\ Chongqing Medical University, Chongqing 400016, P.R. China
}

Received December 19, 2013; Accepted February 28, 2014

DOI: $10.3892 /$ ijmm.2014.1699

\begin{abstract}
Cervical cancer is the principal cause of mortality due to cancer in women worldwide. New predictive markers may increase survival rates by improving the treatment of patients at a high risk for cancer. This study was carried out to investigate the amplification of human telomerase RNA component (hTERC) or/and c-MYC in cervical epithelial exfoliated cells for cervical carcinoma screening. We collected 171 specimens. including speciments from normal cervix, benign lesions, cervical intraepithelial neoplasia (CIN)1, CIN2 and CIN3, or carcinoma in situ, as well as invasive cervical squamous cell carcinoma. Fluorescence in situ hybridization (FISH) was performed to detect alterations in hTERC and c-MYC expression. We analyzed the area under the receiver operating characteristic (ROC) curve (AUC), as well as the sensitivity and specificity of single screening and conjoined screening. There was a trend toward an increasing amplification of 2 genes with the increasing severity of cervical lesions. ROC curve analysis demonstrated that the AUC values of the hTERC gene for the screening of different cervical lesions were $>0.8$. Compared with the hTERC gene, the AUC of the c-MYC gene for the screening of $\geq \mathrm{CIN} 3$ was $>0.8$ and the AUC for the screening of other cervical lesions was $>0.7$. For the screening of cervical lesions above the grade of benign lesions, cytological diagnosis was superior to the gene detection with significant differences. For the screening of cervical lesions $>$ CIN1, there were no statistically significant differences $(\mathrm{P}>0.05)$ between the hTERC gene and cytological diagnosis, whereas the screening results of c-MYC detection and cytological diagnosis differed significantly $(\mathrm{P}<0.05)$. For the screening of cervical lesions $>$ CIN2 or $>$ CIN 3 , the detection of hTERC and c-MYC
\end{abstract}

Correspondence to: Professor Liangdan Tang, Department of Obstetrics and Gynecology, The First Affiliated Hospital of Chongqing Medical University, Chongqing Medical University, 1 You-Yi Road, Yuzhong, Chongqing 400016, P.R. China E-mail: 1584512815@qq.com

Key words: fluorescence in situ hybridization, human telomerase RNA component, c-MYC, cervical cancer screening genes and cytological diagnosis had similar screening results with no statistically significant differences $(\mathrm{P}>0.05)$. In conclusion, using FISH to detect the amplification of hTERC or/and c-MYC on cervical epithelial exfoliated cells may be a useful and specific screening method for precancerous lesions.

\section{Introduction}

Invasive cervical cancer (ICC) is one of the most common types of cancer affecting women worldwide, in both incidence and mortality $(1,2)$. There is a long developmental process between the stages of cervical intraepithelial neoplasia (CIN) to cervical cancer. Early screening and appropriate treatment may prevent the development of CIN; some lesions can be cured completely, and other minor lesions will soon return to normal without treatment (3). Since the introduction of the Papanicolaou test (Pap smear) decades ago, the mortality and morbidity rates for patients with invasive cervical cancer have reduced greatly, particularly in developed countries (4-9). The achievement of Pap smear testing for public health is significant. However, there are still 500,000 cases of cervical cancer and 275,000 cervical cancer deaths among women worldwide, and the incidence rates in developing countries are particularly high (10). The sensitivity of the Pap smear varies substantially in areas with different screening infrastructures (11). In several meta-analyses of the accuracy of Pap smears, the sensitivity ranged from approximately 50 to $80 \%$, but can be as low as $20 \%$ (12-14), which limits the efficacy of cancer detection (15). Over the past decade, the liquid-based Pap test has been introduced to overcome the drawbacks of the conventional Pap smear and has resulted in the reduction of limited and unsatisfactory specimens and in an improvement in the adequacy and detection rates for squamous intraepithelial lesions (16). Due to sampling error, there are still some discrepancies in the interpretation between the cytological and histological diagnoses of the simultaneously sampled smears and biopsy specimens.

Laboratory and epidemiological research has suggested a strong association between HPV infection and cervical cancer (17). However, the discrepancy between high rates of HPV infection and low rates of cervical cancer development among women suggests that additional genetic events are necessary for the progression to a malignant phenotype. Chromosomal instability at a numerical or structural level 
is a hallmark of malignant tumors (18). Indeed, recurrent patterns of chromosomal alterations with specific imbalances that are important for tumor initiation and progression have been revealed in cervical cancer (19). Deletion, duplication and amplification of various genomic regions have been demonstrated in cervical cancer by comparative genomic hybridization and fluorescence in situ hybridization (FISH) methods (20-23). A number of studies have demonstrated frequent gain or loss of several specific chromosomal regions in cervical cancer, e.g., gain of 3q, 5p, 8q, 11q, 17q and 20q and loss of $3 p, 4 p, 5 q$ and $18 q(21,23-28)$. At present, the gene(s) involved in these regions and their biologic functions in this disease have not yet been fully identified.

A proto-oncogene can be abnormally activated and turned into an oncogene; gene amplification is the dominant mechanism for oncogene activation in solid tumors (29). The aberrant activation and/or overexpression of different oncogenes are associated with the development and progression of human tumors (30). MYC genes are key regulators of cell proliferation, and the enhanced expression of MYC genes promotes unrestricted proliferation and contributes to the genesis of most human tumors (31). The amplification and overexpression of the MYC gene have been detected in both cell lines and cervical cancers $(32,33)$. Studies from the US NIH in 2005 for cervical cancer demonstrated that the process of cervical cell variation into cervical cancer is always accompanied with the amplification of the long arm of chromosome $3(34,35)$. The most important genes involved are human telomerase RNA component (hTERC). Sokolova et al (36) assessed biopsy specimens showing high-grade dysplasia and cancer with FISH probes to 35 unique loci and identified 2 loci, the $3 q 26.3$ region (comprising the hTERC gene) and the $8 \mathrm{q} 24$ region (comprising the c-MYC gene), which showed the highest frequency of copy number gains in high-grade dysplasia and cancer. These loci are frequently altered in cervical cancer tumorigenesis $(21,22,28,37)$. Thus, they may be useful markers for the detection of cervical dysplasia and carcinoma.

The FISH technique, which provides an accurate quantification of the signals (2) is relatively simple to operate, has good reproducibility, stability, and has the advantages of good sensitivity and specificity; it does not require cell culture and be used in interphase cells; FISH is easily adaptable to clinical detection.

In this study, the FISH technique was used to detect the amplification of hTERC and c-MYC in cervical epithelial exfoliated cells. Combined with the liquid-based cytology test results of these cases, we assessed the significance of the amplification of hTERC and c-MYC in cervical cancer screening.

\section{Materials and methods}

Cytology specimens. Specimens with an abnormal cytological diagnosis [atypical squamous cells of undetermined significance (ASCUS), atypical squamous cells - cannot exclude high grade squamous intraepithelial lesion (HSIL; ASC-H), low grade squamous intraepithelial lesion (LSIL), HSIL and squamous cell carcinoma (SCC)] or a suspicious diagnosis as described in accordance with the TBS system were included in this study if they had a corresponding biopsy diagnosis [the ThinPrep
Cytology Test (TCT) was used for cytological diagnosis]. Cytology specimens with a cytological diagnosis of 'normal' were also included, but not all of these patient specimens had a corresponding biopsy according to the standard practice of care. We collected exfoliated cells from 171 patients. The distribution of specimens based on the cytology and biopsy classification was as follows: normal uterine cervix specimens with normal cytology and no biopsy available as controls $(n=40)$, benign lesions of the uterine cervix, including squamous metaplasia, chronic cervicitis and atypia of repair, which had a normal to HSIL cytology $(n=24)$, CIN1 cases which had a normal to HSIL cytology $(n=26)$, CIN2 cases which had a normal to HSIL cytology $(n=29)$, and CIN3 or carcinoma in situ cases which had a normal to suspicious carcinoma cytology $(n=36)$. Invasive cervical SCC cases which had a cytology of HSIL to suspicious SCC (ICC), $\mathrm{n}=16$.

Specimens were obtained from the First Affiliated Hospital of Chongqing Medical University (Chongqing, China) between December 2010 and July 2011. Adenocarcinoma specimens were not included. The age of the patients ranged between 22 and 66 years. Exclusion criteria included pregnancy, menstrual period, chronic or acute systemic viral infections, a history of cervical neoplasia, an immunocompromised state, the presence of other cancers, a history of surgery to the uterine cervix, persons had been undergone colposcopic biopsy or cases that had been treated.

A cervical brush (a special Pap Brush; Beijing TCT Medical Technology Company, Ltd., Beijing, China) was used to collect the cervical epithelial exfoliated cells following the manufacturer's instructions. The exfoliated cells were preserved in phosphate-buffered saline at $4^{\circ} \mathrm{C}$ until FISH examination. All the patients were diagnosed and had their specimens banked at the First Affiliated Hospital of Chongqing Medical University. Patients who had low- and high-grade lesions identified by cytology underwent colposcopic cervical biopsy and that sometimes included subsequent conization or major surgery. The final diagnosis was made by tissue-proven pathology rather than cytology, apart from the controls. Controls were recruited from healthy women who underwent routine Pap screening. Informed consent was obtained from all patients and control subjects. The Institutional Review Board of the Peking Union Medical College Hospital as the lead hospital of the project approved this study.

Probe set formulation. The FISH detection kit, including the locus-specific probes 3q26.3 and 8q24 were manufactured using a standard labeling procedure by the Beijing Gold Bodhisattva Ka Medical Technology Co., Ltd, Beijing, China.

Probe group 1: GLP TERC/CSP3. The chromosome probe 3q26.3 (hTERC) was labeled with the Spectrum Red fluorophore. The sequence of the $3 q 26.3$ probe was 5'-CUAACCCUAAC-3'. The CSP3 probe was labeled with the Spectrum Green fluorophore, whose sequence is protected. The CSP3 probe hybridized with the 3p11.1-q11.1 region of the centromere in chromosome 3 , was used as the control probe. There were 2 red and 2 aquamarine hybridization signals in each interphase nucleus of cells which had no amplification of the hTERC gene. At least 2 aquamarine signals and $>2$ red signals should be detected in each interphase nucleus of cells which had an amplification of the hTERC gene. 
Probe group 2: GLP-c-MYC. The probe 8q24 (c-MYC) was labeled with the Spectrum Red fluorophore. The sequence of the $8 \mathrm{q} 24$ probe is protected. There were two red hybridization signals in each interphase nucleus of cells which had no amplification of the c-MYC gene. More than 2 red signals should be detected in each interphase nucleus of cells which had an amplification of the c-MYC gene.

Fish procedures. FISH detected the hTERC and c-MYC genes in cervical epithelial exfoliated cells following the instructions of the FISH detection kit. Approximately 5-10 ml preservation solution containing the exfoliated cells were placed into a suitable centrifuge tube. Following centrifuging at 1,300 rpm for $10 \mathrm{~min}$ and removing the supernatant, the exfoliated cells were supplemeted with $3 \mathrm{ml}$ collagenase $\mathrm{B}$; the suspended cells were then separated, followed by incubation in $37^{\circ} \mathrm{C}$ water for 20-30 min, centrifugation at 1,300 rpm for $10 \mathrm{~min}$, removing the supernatant again, the addition of $5 \mathrm{ml} 37^{\circ} \mathrm{C}$ water, separating the suspended cells, incubation in $37^{\circ} \mathrm{C}$ water for $20 \mathrm{~min}$, the addition of $2 \mathrm{ml}$ stationary liquid, centrifugation at $1,300 \mathrm{rpm}$ for $10 \mathrm{~min}$, removing supernatant, the addition of $5 \mathrm{ml}$ stationary liquid, centrifugation at 1,300 rpm for $10 \mathrm{~min}$, and repeating the section as described above, and finally removing the supernatant, followed by the addition of appropriate stationary liquid, separating the suspended cells, and dropping to the glass slides followed by air drying overnight at room temperature. The following day, the slides were soaked twice in $2 \mathrm{X} \mathrm{SSC} \mathrm{(PH} \mathrm{7.0)} \mathrm{at} \mathrm{room} \mathrm{temperature} \mathrm{for}$ $5 \mathrm{~min}$ and then soaked in $100 \mathrm{mmol} / \mathrm{l} \mathrm{HCl}$ at room temperature for $10 \mathrm{~min}$. The slides were then incubated in pepsin (approximately $0.02 \mathrm{mg} / \mathrm{ml}$ in $10 \mathrm{mmol} / 1 \mathrm{HCl}$ ) at $37^{\circ} \mathrm{C}$ for $10 \mathrm{~min}$. Mild pepsin digestion was performed to increase the penetration of the probes. The slides were then soaked twice in $2 \mathrm{X}$ SSC at room temperature for $5 \mathrm{~min}$, fixed in $1 \%$ neutral-buffered formalin at room temperature for $5 \mathrm{~min}$, dehydrated in an ethanol series of 70,85 and $100 \%$ for $3 \mathrm{~min}$ in each solution and air-dried. The probe mixture was then applied, and the slides were coverslipped and sealed with rubber cement. The slides with probe mix were co-denatured at $77^{\circ} \mathrm{C}$ for $5 \mathrm{~min}$, followed by hybridizing at $42^{\circ} \mathrm{C}$ for $16-18 \mathrm{~h}$.

Following hybridization and the removal of the coverslips, the slides were washed in 3 bottles of $2 \mathrm{X} \mathrm{SSC} / 50 \%$ formamide solution for $10 \mathrm{~min}$ in each bottle, in $2 \mathrm{X} \mathrm{SSC}$ for $10 \mathrm{~min}$, in $0.1 \%$ Nonidet P-40/2X SSC for $5 \mathrm{~min}$, in $70 \%$ ethanol for $3 \mathrm{~min}$. Following stringent washing with a series of solution, the slides followed by air drying were applied with the anti-fade solution containing the nuclear counterstain 4,6-diamidino2-phenylindole (DAPI), and the slides were coverslipped. After being placed back in a black box for 10-20 min, the slides were observed under a fluorescence microscope (Olympus).

Signal scoring and evaluation. Each sample had 2 slides and each slide was detected by FISH with 1 probe group. All the slides were analyzed under a fluorescence microscope using $\mathrm{x} 10, \mathrm{x} 40$ and $\mathrm{x} 100$ magnification equipped with the corresponding wavelength filter, a CCD camera and an imagecapturing and analyzing system. A magnification of x10 to was used to find the cell region in slide; $x 40$ magnification was used to scan the entire hybrid area and observe the quality of the specimens, and x100 magnification was used for the evaluation of signal scoring. In most cases, the entire surface area of each slide was analyzed. However, in cases involving a large number of cells per slide, only the first 100 cells were analyzed. In these cases, the estimated number of cells on the entire slide was extrapolated from the percentage of surface area enumerated after enumerating 100 cells. The exact copy number of the signals per nucleus was recorded, and at least 100 nuclei/sample were analyzed. Only satisfactory samples with $\geq 3$ copies/nucleus of the given genes in $>75 \%$ of the counted cells were considered to contain gene amplification.

The images in Fig. 1 consist of chromosome probe GLP TERC/CSP3 visualized red and aquamarine hybridization signals, and chromosome probe GLP-c-MYC visualized red hybridization signals. A cell has no amplification of the hTERC gene with only 2 red and 2 aquamarine hybridization signals in each interphase nucleus (Fig. 1D). A cell has amplification of the hTERC gene with at least 2 aquamarine signals and $>2$ red hybridization signals in each interphase nucleus (Fig. 1B). A cell has no amplification of the c-MYC gene with only 2 red hybridization signals in each interphase nucleus (Fig. 1C). A cell has amplification of the c-MYC gene with $>2$ red hybridization signals in each interphase nucleus (Fig. 1A).

Twenty cases of the control group were first examined by FISH. One hundred nuclei/sample were analyzed, and the percentage of cells that had amplification of the hTERC or c-MYC gene in the enumerated cells was counted and used to establish threshold values as follows: threshold $=$ mean $(M)+3 \times$ standard deviation $(\mathrm{SD})$. If the proportion of the detection was more than the threshold, then the sample was evaluated as positive; if it was less than the threshold was negative; if it was equal to the threshold value then the number of cells observed needed to be increased. In this study, the threshold value was 5 for enumeration.

Data analysis. The enumeration results were analyzed using SAS9.2 statistical software for rank sum test and MedCalc 12.3.0 statistical software for the receiver operating characteristic (ROC) curve analyses. P-values $<0.05$ were considered to indicate statistically significant differences.

\section{Results}

FISH was successively performed on 171 cases. The distribution of the specimens based on the cytology and biopsy classification is shown in Table I.

Amplification of hTERC and c-MYC. The results of FISH analysis on 171 cases were sorted as positive or negative statistical results using the threshold (gene detection result $\geq 5$ ) (Table II). For 2 genes (hTERC and c-MYC), there was a trend toward increasing amplification with the increasing severity of the cervical squamous lesions. Amplification was detected for the hTERC gene in 0 of the 40 controls $(0 \%), 6$ of the 24 cervicitis (25\%), 6 of the 26 CIN1 (23.08\%), 15 of the 29 CIN2 (51.72\%), 26 of the 36 CIN3 (72.22\%) and in 14 of the 16 ICC samples (87.50\%) (Table II). Furthermore, as shown in Table II, amplification for the c-MYC gene was detected in 0 of the 40 controls ( $0 \%), 4$ of the 24 cervicitis (16.67\%), 5 of the 26 CIN1 (19.23\%), 11 of the 29 CIN2 (37.93\%), 22 of the 

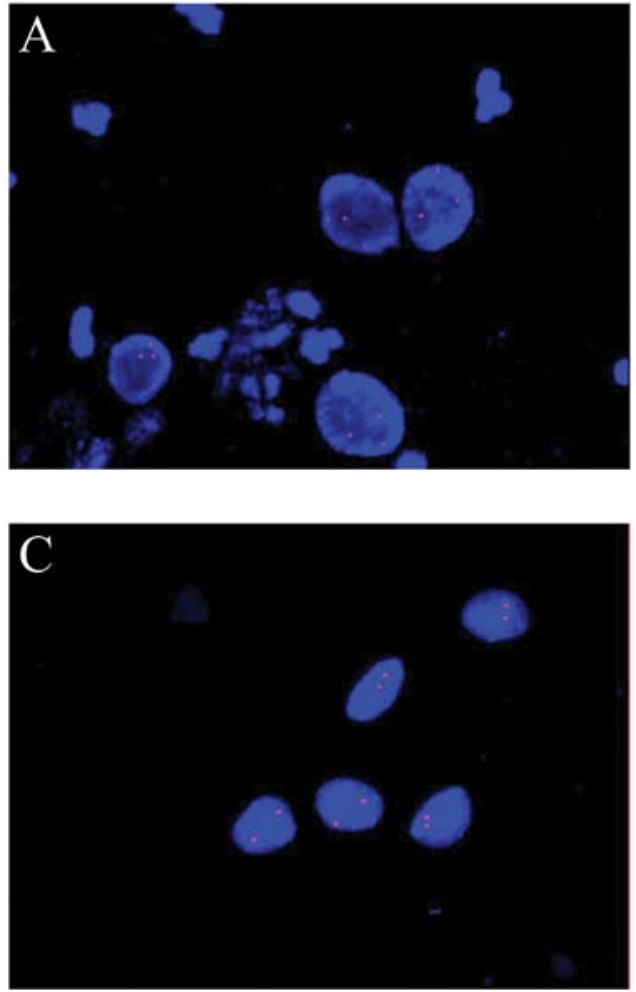
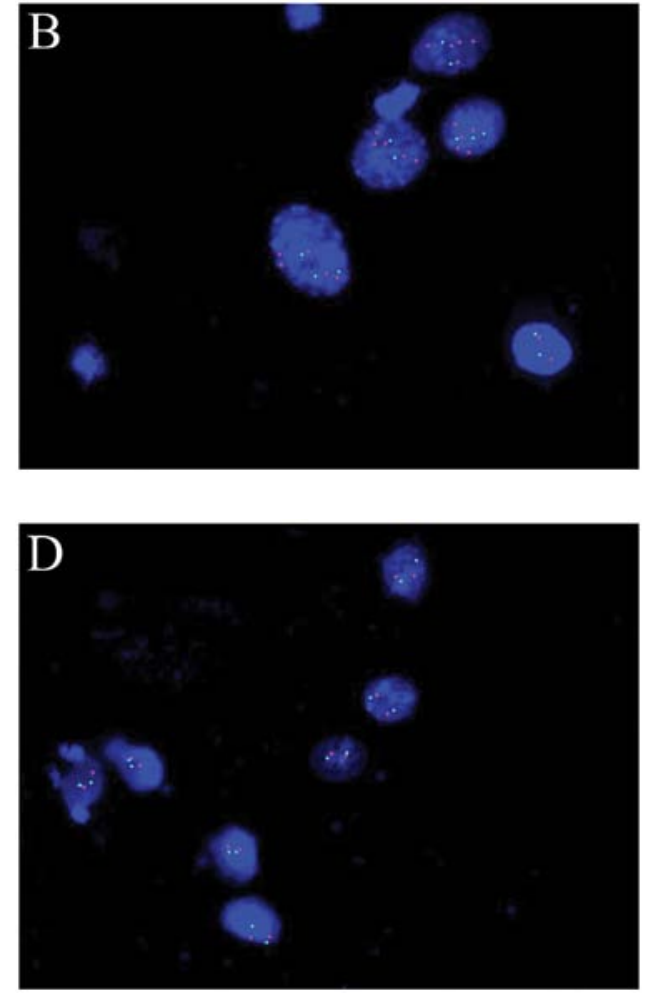

Figure 1. Representative images of chromosome probes 3q26.3 [human telomerase RNA component (hTERC)] and 8q24 (c-MYC) signals observed in cervical epithelial cells after fluorescence in situ hybridization (FISH) assay. Each individual signal for the hTERC and c-MYC probes constitutes the respective gene. The images consist of chromosome probe GLP TERC/CSP3 visualized red and green hybridization signals, and chromosome probe GLP-c-MYC visualized red hybridization signals. (A) Represents a cell that has amplification of the c-MYC gene. (B) Represents a cell that has amplification of the hTERC gene. (C) Represents a cell that has no amplification of the c-MYC gene. (D) Represents a cell that has no amplification of the hTERC gene.

Table I. Distribution of specimens based on the cytology and biopsy classification.

\begin{tabular}{|c|c|c|c|c|c|c|c|}
\hline \multirow[b]{2}{*}{ Cytology } & \multirow[b]{2}{*}{ Normal } & \multicolumn{5}{|c|}{ Biopsy } & \multirow[b]{2}{*}{ Total } \\
\hline & & Benign lesion & CIN1 & CIN2 & CIN3 & ICC & \\
\hline NILM & 40 & 8 & 4 & 2 & 1 & 0 & 55 \\
\hline ASCUS & 0 & 3 & 9 & 2 & 3 & 0 & 17 \\
\hline ASC-H & 0 & 4 & 3 & 2 & 2 & 0 & 11 \\
\hline LSIL & 0 & 6 & 4 & 5 & 0 & 0 & 15 \\
\hline HSIL & 0 & 3 & 5 & 18 & 28 & 10 & 64 \\
\hline $\mathrm{SCC}$ & 0 & 0 & 1 & 0 & 2 & 6 & 9 \\
\hline Total & 40 & 24 & 26 & 29 & 36 & 16 & 171 \\
\hline
\end{tabular}

CIN, cervical intraepithelial neoplasia; ASCUS, atypical squamous cell of undetermined significance; ICC, invasive cervical cancer; NILM, negative for intraepithelial or malignant cervical cytology; ASC-H atypical squamous cells - cannot exclude HSIL; LSIL, low grade squamous intraepithelial lesion; HSIL, high grade squamous intraepithelial lesion; SCC, squamous cell carcinoma.

36 CIN3 (61.11\%) and in 14 of the 16 ICC (87.50\%) samples. hTERC was more frequently amplified than c-MYC. When a case had an amplification of the c-MYC gene, it generally had an amplification of the hTERC gene.

In the enumerated cells of each sample, the percentage of cells with an abnormal amplification of the hTERC and c-MYC genes is termed as the abnormal amplification rate for short. The respective abnormal amplification rates of the above 2 genes in different histological lesions were subjected to the rank-sum test. Multiple comparisons were performed using the Kruskal-Wallis rank-sum test. The results revealed that multiple comparisons of the abnormal amplification rates of the hTERC and c-MYC genes in the different histological lesions all had statistical significance $(\mathrm{P}<0.001)$ (Table III). Therefore, a pairwise comparison was conducted using the Mann-Whitney rank-sum test. The hTERC probe revealed significant differences of several groups except between benign lesions and CIN1, CIN2 and CIN3. Similarly, the 

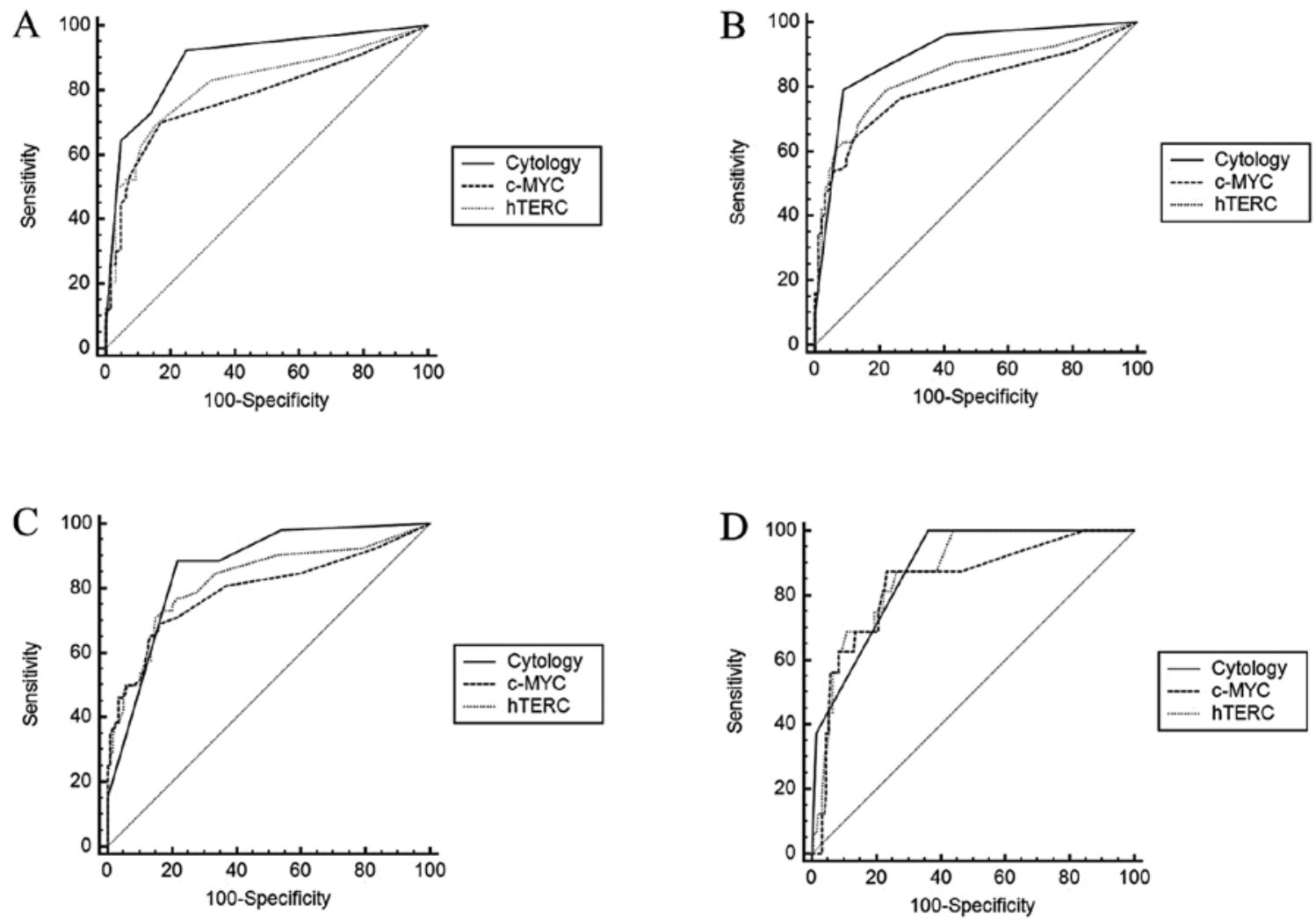

Figure 2. Comparison of receiver operating characteristic (ROC) curve analysis for human telomerase RNA component (hTERC), c-MYC and cytology in discriminating between different types or worse of certain cervical intraepithelial neoplasia (CIN). (A) Represents type over benign lesion. (B) Represents type over CIN1. (C) Represents type over CIN2. (D) Represents type over CIN3.

Table II. Detection of hTERC and c-MYC in 6 groups [n (\%)].

\begin{tabular}{lcccc}
\hline Group & N & \multicolumn{1}{c}{ hTERC } & \multicolumn{1}{c}{ c-MYC } & \multicolumn{1}{c}{$\begin{array}{l}\text { Double- } \\
\text { positive }\end{array}$} \\
\hline Normal & 40 & $0(0)$ & \multicolumn{1}{l}{$0(0)$} & $0(0)$ \\
Benign lesion & 24 & $6(25.00)$ & $4(16.67)$ & $4(16.67)$ \\
CIN1 & 26 & $6(23.08)$ & $5(19.23)$ & $5(19.23)$ \\
CIN2 & 29 & $15(51.72)$ & $11(37.93)$ & $11(37.93)$ \\
CIN3 & 36 & $26(72.22)$ & $22(61.11)$ & $22(61.11)$ \\
ICC & 16 & $14(87.50)$ & $14(87.50)$ & $14(87.50)$ \\
\hline
\end{tabular}

hTERC, human telomerase RNA component; N, number of cases; CIN, cervical intraepithelial neoplasia; ICC, invasive cervical cancer.

c-MYC probe exhibited significant differences of several groups except between benign lesions and CIN1, CIN2 and CIN3, CIN3 and cervical cancer.

Associations between gene amplification and clinical diagnosis. On the basis of the percentage of cells that had gene amplification for the chromosome probe markers, 3q26.3 (hTERC) or 8q24 (c-MYC), in the enumerated cells per sample, ROC curves were generated to confirm the accuracy of diagnosis of each mean (Fig. 2). Sensitivity and specificity were computed for each mean at the best. The area under the
Table III. Comparison of the amplification rate for hTERC and c-MYC in the different groups $(\%, \mathrm{M})$.

\begin{tabular}{lccc}
\hline Group & N & hTERC & c-MYC \\
\hline Normal & 40 & 1 & 1 \\
Benign lesion & 24 & 1 & 2 \\
CIN1 & 26 & 2 & 2.5 \\
CIN2 & 29 & 8 & 4 \\
CIN3 & 36 & 20 & 17 \\
ICC & 16 & 49.5 & 52 \\
P-value & & $<0.001$ & $<0.001$
\end{tabular}

hTERC, human telomerase RNA component; N, number of cases; CIN, cervical intraepithelial neoplasia; ICC, invasive cervical cancer.

ROC curve (AUC) of each grade was calculated for the diagnosis of a certain CIN type or worse with certain critical value (Tables IV-VI). According to the AUC values calculated, the comparison of hTERC, c-MYC and cytology in discriminating certain a CIN type or worse is shown in Table VII. Cytological diagnosis involved digitization as follows. negative for intraepithelial or malignant (NILM) cervical cytology, 0; ASCUS/ASC-H, 1; LSIL, 2; HSIL, 3; and SCC, 4.

The performance of combined testing also was calculated. According to AUC values calculated, the current results indicated that the combined detection of the hTERC and 
Table IV. Comparison of hTERC detection for the screening of different cervical lesions.

\begin{tabular}{lcccccr}
\hline Screening level & Critical value (\%) & SEN & SPE & AUC & 95\% CI & P-value \\
\hline$>$ Benign lesion & $>2$ & 69.16 & 84.37 & 0.817 & $0.751-0.872$ & $<0.0001$ \\
& $>4$ & 67.90 & 86.67 & & & \\
$>$ CIN1 & $>2$ & 79.01 & 77.78 & 0.838 & $0.774-0.889$ & $<0.0001$ \\
& $>4$ & 57.01 & 90.62 & & & $<0.0001$ \\
$>$ CIN2 & $>14$ & 71.15 & 84.87 & 0.825 & $0.759-0.878$ & $<0.0001$ \\
$>$ CIN3 & $>14$ & 87.50 & 73.55 & 0.873 & $0.813-0.919$ & \\
\hline
\end{tabular}

SEN, sensitivity; SPE, specificity; AUC, area under the ROC curve; CI, confidence interval; hTERC, human telomerase RNA component; CIN, cervical intraepithelial neoplasia.

Table V. Comparison of c-MYC detection for the screening of different cervical lesions.

\begin{tabular}{|c|c|c|c|c|c|c|}
\hline Screening level & Critical value (\%) & SEN & SPE & AUC & $95 \% \mathrm{CI}$ & P-value \\
\hline \multirow[t]{2}{*}{ >Benign lesion } & $>2$ & 70.09 & 82.81 & 0.780 & $0.711-0.840$ & $<0.0001$ \\
\hline & $>4$ & 48.60 & 93.75 & & & \\
\hline \multirow[t]{2}{*}{$>\mathrm{CIN} 1$} & $>3$ & 64.20 & 87.78 & 0.799 & $0.732-0.857$ & $<0.0001$ \\
\hline & $>4$ & 58.02 & 90.00 & & & \\
\hline$>\mathrm{CIN} 2$ & $>6$ & 69.23 & 84.03 & 0.794 & $0.725-0.852$ & $<0.0001$ \\
\hline$>\mathrm{CIN} 3$ & $>10$ & 87.50 & 76.77 & 0.840 & $0.776-0.891$ & $<0.0001$ \\
\hline
\end{tabular}

SEN, sensitivity; SPE, specificity; AUC, area under the ROC curve; CI, confidence interval; CIN, cervical intraepithelial neoplasia.

Table VI. Comparison of cytological detection for the screening of different cervical lesions.

\begin{tabular}{lccccrr}
\hline Screening level & Critical value & SEN & SPE & AUC & 95\% CI & P-value \\
\hline Benign lesion & $>0$ & 92.52 & 75.00 & 0.894 & $0.838-0.936$ & $<0.0001$ \\
CIN1 & $>2$ & 79.01 & 91.11 & 0.900 & $0.845-0.941$ & $<0.0001$ \\
CIN2 & $>2$ & 88.46 & 78.15 & 0.863 & $0.802-0.911$ & $<0.0001$ \\
CIN3 & $>2$ & 100.00 & 63.87 & 0.881 & $0.822-0.925$ & $<0.0001$ \\
\hline
\end{tabular}

SEN, sensitivity; SPE, specificity; AUC, area under the ROC curve; CI, confidence interval; CIN, cervical intraepithelial neoplasia.

Table VII. Comparison of hTERC, c-MYC and cytology in discriminating between the different types [or worse $(>)$ ] of cervical intraepithelial neoplasia.

\begin{tabular}{lllll}
\hline & \multicolumn{4}{c}{ P-value } \\
\cline { 2 - 5 } Comparison & $>$ BEN & $>$ CIN1 & $>$ CIN2 & $>$ CIN3 \\
\hline Cytology and c-MYC & 0.0015 & 0.0050 & 0.0994 & 0.4259 \\
Cytology and hTERC & 0.0210 & 0.0520 & 0.3023 & 0.8491 \\
c-MYC and hTERC & 0.2035 & 0.1698 & 0.2973 & 0.4314 \\
\hline
\end{tabular}

hTERC, human telomerase RNA component; BEN, benign; CIN, cervical intraepithelial neoplasia.
c-MYC genes in cervical epithelial exfoliated cells by the FISH technique could better discriminate between the CIN1 and worse group (Table VIII). Their sensitivity and specificity are shown in Table VIII in detail. ROC curve analysis demonstrated that the sensitivity and specificity for detecting CIN2+ lesions (CIN2 or worse) were 57.01 and 90.62, respectively for testing for hTERC alone; 58.02 and 90, respectively for testing for c-MYC alone; and 60.49 and 92.22, respectively for the combined testing of hTERC and c-MYC.

\section{Discussion}

Cervical cancer is the principal cause of mortality due to cancer in women worldwide. The 5-year survival rate ranges from 
Table VIII. Comparison of estimated SEN, SPE with hTERC, c-MYC and cytology in discriminating between different types of cervical intraepithelial neoplasia lesions.

\begin{tabular}{lcccc}
\hline & SEN & SPE & AUC & 95\% CI \\
\hline hTERC & 57.01 & 90.62 & 0.838 & $0.774-0.889$ \\
c-MYC & 58.02 & 90.00 & 0.799 & $0.732-0.857$ \\
Cytology & 79.01 & 91.11 & 0.900 & $0.845-0.941$ \\
hTERC and c-MYC & 60.49 & 92.22 & 0.764 & $0.693-0.825$ \\
hTERC and cytology & 65.43 & 86.67 & 0.760 & $0.689-0.822$ \\
c-MYC and cytology & 58.02 & 92.22 & 0.751 & $0.679-0.814$ \\
hTERC and c-MYC and cytology & 58.02 & 92.22 & 0.751 & $0.679-0.814$ \\
\hline
\end{tabular}

SEN, sensitivity; SPE, specificity; AUC, area under the ROC curve; CI, confidence interval; hTERC, human telomerase RNA component.

$15-80 \%$, depending on the extent of the disease (38). Novel predictive markers may increase survival rates by improving the treatment of patients at high risk for cancer. Pre-invasive disease can be detected by cervical cytology. All currently available cytology technologies rely on the visual analysis of exfoliated cells from the uterine cervix. Improvement of conventional cytological screening has been proposed by the introduction of molecular-based markers applied to liquidbased cytology (LBC), the suspension of cells collected from the cervix (39).

The distribution of specimens based on the cytological and histological classification varied: some cases had normal cytology and normal biopsy, some cases had ASCUS cytology and CIN2/3 biopsy, some cases had LSIL cytology and normal biopsy, some cases had LSIL cytology and CIN1 biopsy, some cases had LSIL cytology and CIN2/3 biopsy, and some cases had HSIL cytology and CIN2/3 biopsy, and so on. Not every abnormal cytology case had pathological abnormality. For example, the CIN2 group contained cases from normal to HSIL cytology in this study.

The development of cervical carcinoma is closely associated with HPV infection. However, other genetic alterations also play an important role $(17,19,36,42)$. Previous studies have shown that the detection of telomerase activity in the cervix may provide information on cervical carcinogenesis and may be a marker to monitor CIN transition $(2,21,23,25,36)$. A systematic review was performed to evaluate the telomerase test (telomerase repeated amplification protocol) for the diagnosis of cervical lesions and compare it to paraffin-embedded sections as the diagnostic standard (40). Data suggest that telomerase may activate an early event in cervical carcinogenesis that may be associated with the initiation and progression of cervical lesions (40). Invasive cervical carcinomas almost invariably carry extra copies of the chromosome arm 3q, resulting in a gain of the human telomerase gene (TERC). This provides the rationale for the development of a multicolor FISH probe set as a diagnostic tool for the direct detection of TERC gains in Pap smears. The study by Heselmeyer-Haddad et al (35) showed that CIN2 and CIN3 lesions can be distinguished from normal samples, ASCUS and CIN1, with a sensitivity and specificity exceeding $90 \%$, independent of the cytomorphological assessment; a $3 \mathrm{q}$ gain is required for the transition from CIN1/ CIN2 to CIN3 and it predicts progression. The sensitivity of the research for predicting the progression from CIN1/CIN2 to CIN3 was $100 \%$ and the specificity, i.e., the prediction of regression, was $70 \%$. Thus the detection of $3 q$ gain and amplification of TERC in routinely collected Pap smears can assist in identifying low-grade lesions with a high progression risk and in decreasing false-negative cytological screenings. The study by Li et al (41) showed that the amplification of hTERC was $6.06 \%$ in normal or cases of inflammation, $10.00 \%$ in CIN1, 66.67\% in CIN2, $72.50 \%$ in CIN3, and $100.00 \%$ in carcinoma cases, with a significant difference between the low- ( $\leq$ CIN1 or $\leq$ low-grade squamous intraepithelial lesion) and high-grade ( $\geq$ CIN2 or $\geq$ atypical squamous cells in which high-grade squamous intraepithelial lesion cannot be excluded) cervical lesions $(\mathrm{P}<0.001)$; the hTERC amplification rate was consistent with the abnormal rates of cytological and histological diagnoses. Thus, using FISH to detect the amplification of hTERC may be a useful and specific screening method in cervical cancer and precancerous lesions. Our study indicated that the amplification of hTERC was $9.38 \%$ in normal or cases of inflammation, $23.08 \%$ in CIN1, $51.72 \%$ in CIN2, $72.22 \%$ in CIN3, and $87.50 \%$ in carcinoma cases. ROC curve analysis demonstrated that the AUC values of hTERC gene detection for screening of cervical cancer of different grades were $>0.8$, suggesting that excellent diagnostic results were obtained with this indicator. When the abnormal amplification rate of the hTERC gene was $>4$, cervical lesions of $\leq \mathrm{CIN} 1$ and $\geq \mathrm{CIN} 2$ were better distinguished (sensitivity, 57.01\%; specificity, $90.62 \%$ ); when the abnormal amplification rate of the hTERC gene was $>14$, cervical lesions of $\geq \mathrm{CIN} 2$ were better diagnosed (sensitivity, 71.15\%; specificity, 84.87\%).

Zhang et al (2) analyzed copy number alterations of several oncogene loci in a panel of 84 cervical tumors. c-MYC at $8 q 24$ was included. The amplification of c-MYC was detected in $25 \%$ of tumors, using interphase FISH. The increased protein expression of c-MYC was observed in tumors with the corresponding gene amplification. c-MYC may have critical biological impact on the development and progression of carcinoma of the uterine cervix. In an internal study, Sokolova et al (36) assessed FISH probes to the 3q26 and the $8 \mathrm{q} 24$ regions on a new set of 100 biopsy cases. Experimentation revealed that the $3 q 26$ and the $8 q 24$ regions had the highest frequency of copy number gains in samples with high-grade dysplasia and cancer. Specifically, the 3 q26 probe was positive 
in $100 \%$ of cancer specimens, $90 \%$ of CIN3 specimens, $78 \%$ of CIN2 specimens, $26 \%$ of CIN1 specimens, and $0 \%$ of normal specimens. The $8 \mathrm{q} 24$ probe was positive in 100, 95, 96, 26 and $5 \%$ of cancer, CIN3, CIN2, CIN1 and normal cases, respectively. The increasing trend was in agreement with our findings. Our study indicated that the amplification of c-MYC was $6.25 \%$ in normal or cases of inflammation, $19.23 \%$ in CIN1, 37.93\% in CIN2, $61.11 \%$ in CIN3 and $87.50 \%$ in carcinoma cases. ROC curve analysis demonstrated that the AUC value of c-MYC gene detection for the screening of cervical lesions $\geq \mathrm{CIN} 3$ was $>0.8$ and the AUC values for screening of cervical lesions of other grades were $>0.7$, indicating the moderate diagnostic results of this indicator. When the abnormal amplification rate of c-MYC was $>10$, cervical lesions $\geq \mathrm{CIN} 3$ were better diagnosed (sensitivity, $87.50 \%$; specificity, 76.77\%). Furthermore, our study revealed that using FISH to detect the amplification of hTERC had a bigger AUC for the diagnoses of different cervical lesions than the detection of c-MYC.

In the current study, the screening results of cervical lesions by hTERC gene detection, c-MYC gene detection, and cytological diagnosis alone were compared. For the screening of cervical lesions above the grade of benign lesions, cytological diagnosis was superior to the gene detection with significant differences in the diagnostic results from the 2 gene detection methods. For the screening of cervical lesions >CIN1, the greatest AUC value was obtained in cytological diagnosis, followed by hTERC detection; however, there were no statistically significant differences $(\mathrm{P}>0.05)$ observed in the screening results between hTERC gene detection and cytological diagnosis, whereas the screening results of c-MYC detection and cytological diagnosis were significantly different $(\mathrm{P}<0.05)$. For the screening of cervical lesions $>\mathrm{CIN} 2$, the detection of hTERC and c-MYC genes and cytological diagnosis had similar screening results with no statistically significant differences $(\mathrm{P}>0.05)$.

In addition, the screening results of cervical lesions by a combination of hTERC gene detection, c-MYC gene detection, and/or cytological diagnosis were examined. For cervical lesions $\geq \mathrm{CIN} 1$, the combined detection of hTERC and c-MYC genes improved the specificity, but decreased the sensitivity of screening; likewise, the combination of the detection of 2 genes and the cytological method improved the specificity, but markedly decreased the sensitivity of screening. For cervical lesions $\geq C I N 2$, the combined detection of hTERC and c-MYC genes increased both the specificity and sensitivity of screening. For cervical lesions $\geq \mathrm{CIN} 3$ and cervical cancer, different combinations of the detection of 2 genes and the cytological method did not markedly improve the specificity or sensitivity of screening.

In the present study, there were a significant number of cases with marked discrepancies in the interpretation between the cytological and histological diagnoses of the simultaneously sampled Pap smears and biopsy specimens. These discrepancies between the cytological and histological diagnoses are likely to be attributed to sampling error rather than interpretation error. Although the amplification of hTERC or/and c-MYC results in the discrepant cases also showed discordance between the liquid-based cytology Pap smears and biopsy specimens, our data reveals that using FISH to detect the amplification of hTERC or/and c-MYC on cervical epithelial exfoliated cells may be as a useful and specific screening method in pre-cancerous lesions and may be helpful in arbitrating some diagnostic disagreements, although obtaining additional biopsies or Pap smears may eliminate these discrepancies.

\section{References}

1. Jemal A, Bray F, Center MM, Ferlay J, et al: Global cancer statistics. CA Cancer J Clin 61: 69-90, 2011.

2. Zhang A, Månér S, Betz R, Angström T, Stendahl U, Bergman F, Zetterberg A and Wallin KL: Genetic alterations in cervical carcinomas: frequent low-level amplifications of oncogenes are associated with human papillomavirus infection. Int J Cancer 101: 427-433, 2002.

3. Wright TC Jr, Massad LS, Dunton CJ, et al: 2006 consensus guidelines for the management of women with abnormal cervical screening tests. J Low Genit Tract Dis 11: 201-222 ,2007.

4. Christopherson WM, Parker JE, Mendez WM and Lundin FE Jr: Cervix cancer death rates and mass cytologic screening. Cancer 26: 808-811, 1970.

5. Quinn M, Babb P, Jones J and Allen E: Effect of screening on incidence of and mortality from cancer of cervix in England: evaluation based on routinely collected statistics. BMJ 318: 904-908, 1999

6. Pak SC, Martens M, Bekkers R, et al: Pap smear screening history of women with squamous cell carcinoma and adenocarcinoma of the cervix. Aust N Z J Obstet Gynaecol 47: 504-507, 2007.

7. Mählck CG, Jonsson H and Lenner P: Pap smear screening and changes in cervical cancer mortality in Sweden. Int J Gynaecol Obstet 44: 267-272, 1994.

8. Liu S, Semenciw R, Probert A and Mao Y: Cervical cancer in Canada: changing patterns in incidence and mortality. Int $\mathbf{J}$ Gynecol Cancer 11: 24-31, 2001.

9. Etzioni R, Urban N, Ramsey S, et al: The case for early detection. Nat Rev Cancer 3: 243-252, 2003.

10. Parkin DM, Bray F, Ferlay $\mathrm{J}$ and Pisani P: Estimating the world cancer burden: Globocan 2000. Int J Cancer 94: 153-156, 2001.

11. Cuzick J, Szarewski A, Cubie H, et al: Management of women who test positive for high-risk types of human papillomavirus: the HART study. Lancet 362: 1871-1876, 2003.

12. Fahey MT, Irwig L and Macaskill P: Meta-analysis of Pap test accuracy. Am J Epidemiol 141: 680-689, 1995.

13. Koliopoulos G, Arbyn M, Martin-Hirsch P, Kyrgiou M, Prendiville W and Paraskevaidis E: Diagnostic accuracy of human papillomavirus testing in primary cervical screening: a systematic review and meta-analysis of non-randomized studies. Gynecol Oncol 104: 232-246, 2007.

14. Mayrand MH, Duarte-Franco E, Rodrigues I, et al: Human papillomavirus DNA versus Papanicolaou screening tests for cervical cancer. N Engl J Med 357: 1579-1588, 2007.

15. Nanda K, McCrory DC, Myers ER, et al: Accuracy of the Papanicolaou test in screening for and follow-up of cervical cytologic abnormalities: a systematic review. Ann Intern Med 132: 810-819, 2000.

16. Guidos BJ and Selvaggi SM: Use of the Thin Prep Pap Test in clinical practice. Diagn Cytopathol 20: 70-73, 1999.

17. Zur Hausen H: Papillomaviruses causing cancer: evasion from hostcell control in early events in carcinogenesis. J Natl Cancer Inst 92: 690-698, 2000.

18. Duensing S and Münger K: Mechanisms of genomic instability in human cancer: insights from studies with human papillomavirus oncoproteins. Int J Cancer 109: 157-162, 2004.

19. Lazo PA: The molecular genetics of cervical carcinoma. Br J Cancer 80: 2008-2018, 1999.

20. Kurtycz D, Nuñez M, Arts T, Bauman C, Harris C, Inhorn S and Meisner L: Use of fluorescent in-situ hybridization to detect aneuploidy in cervical dysplasia. Diagn Cytopathol 15: 46-51, 1996.

21. Heselmeyer K, Macville M, Schröck E, Blegen H, Hellström AC, Shah K, Auer G and Ried T: Advanced-stage cervical carcinomas are defined by a recurrent pattern of chromosomal aberrations revealing high genetic instability and a consistent gain of chromosome arm 3q. Genes Chromosomes Cancer 19: 233-240, 1997.

22. Thein A, Trková M, Fox M and Parrington J: The application of comparative genomic hybridization to previously karyotyped cervical cancer cell lines. Cancer Genet Cytogenet 116: 59-65, 2000 . 
23. Kirchhoff M, Rose H, Petersen BL, Maahr J, Gerdes T, Lundsteen C, Bryndorf T, Kryger-Baggesen N, Christensen L, Engelholm SA and Philip J: Comparative genomic hybridization reveals a recurrent pattern of chromosomal aberrations in severe dysplasia/carcinoma in situ of the cervix and in advanced-stage cervical carcinoma. Genes Chromosomes Cancer 24: 144-150, 1999.

24. Mullokandov MR, Kholodilov NG, Atkin NB, Burk RD, Johnson AB and Klinger HP: Genomic alterations in cervical carcinoma: losses of chromosome heterozygosity and human papilloma virus tumor status. Cancer Res 56: 197-205, 1996.

25. Heselmeyer K, Schröck E, du Manoir S, Blegen H, Shah K, Steinbeck R, Auer G and Ried T: Gain of chromosome 3q defines the transition from severe dysplasia to invasive carcinoma of the uterine cervix. Proc Natl Acad Sci USA 93: 479-484, 1996.

26. Kersemaekers AM, van de Vijver MJ, Kenter GG and Fleuren GJ: Genetic alterations during the progression of squamous cell carcinomas of the uterine cervix. Genes Chromosomes Cancer 26: 346-354, 1999.

27. Dellas A, Torhorst J, Jiang F, Proffitt J, Schultheiss E, Holzgreve W, Sauter G, Mihatsch MJ and Moch H: Prognostic value of genomic alterations in invasive cervical squamous cell carcinoma of clinical stage IB detected by comparative genetic hybridization. Cancer Res 59: 3475-3479, 1999.

28. Hidalgo A, Schewe C, Petersen S, Salcedo M, Gariglio P, Schlüns K, Dietel $M$ and Petersen I: Human papilloma virus status and chromosomal imbalances in primary cervical carcinomas and tumor cell lines. Eur J Cancer 36: 542-548, 2000.

29. Todd R and Wong DT: Oncogenes. Anticancer Res 19: 4729-4746, 1999.

30. Bishop JM: Molecular themes in oncogenesis. Cell 64: 235-248, 1991.

31. Adhikary S and Eilers M: Transcriptional regulation and transformation by Myc proteins. Nat Rev Mol Cell Biol 6: 635-645, 2005.

32. Couturier J, Sastre-Garau X, Schneider-Maunoury S, Labib A and Orth G: Integration of papillomavirus DNA near myc genes in genital carcinomas and its consequences for proto-oncogene expression. J Virol 65: 4534-4538, 1991.
33. Sastre-Garau X, Favre M, Couturier J and Orth G: Distinct patterns of alteration of myc genes associated with integration of human papillomavirus type 16 or type 45 DNA in two genital tumors. J Gen Virol 81: 1983-1993, 2000.

34. Umayahara K, Hirai Y, Sugiyama Y, et al: Genetic alterations during the progression of early cervical neoplasm. Proc Amer Assoc Cancer Res 46: 5743, 2005.

35. Heselmeyer-Haddad K, Sommerfeld K, White NM, et al: Genomic amplification of the human telomerase gene (TERC) in pap smears predicts the development of cervical cancer. Am J Pathol 166: 1229-1238, 2005.

36. Sokolova I, Algeciras-Schimnich A, Song M, et al: Chromosomal biomarkers for detection of human papillomavirus associated genomic instability in epithelial cells of cervical cytology specimens. J Mol Diagn 9: 604-611, 2007.

37. Matthews CP, Shera KA and McDougall JK: Genomic changes and HPV type in cervical carcinoma. Proc Soc Exp Biol Med 223 316-321, 2000

38. Widschwendter A, Ivarsson L, Blassnig A, et al: CDH1 and CDH13 methylation in serum is an independent prognostic marker in cervical cancer patients. Int J Cancer 109: 163-166, 2004.

39. Apostolidou S, Hadwin R, Burnell M, et al: DNA methylation analysis in liquid-based cytology for cervical cancer screening. Int J Cancer 125: 2995-3002, 2009.

40. Rosa MI, Medeiros LR, Bozzetti MC, et al: Accuracy of telomerase in cervical lesions: a systematic review. Int J Gynecol Cancer 17: 1205-1214, 2007.

41. Li Y, Ye F, Lü WG, et al: Detection of human telomerase RNA gene in cervical cancer and precancerous lesions: comparison with cytological and human papillomavirus DNA test findings. Int J Gynecol Cancer 20: 631-637, 2010.

42. Walboomers JM, Jacobs MV, Manos MM, et al: Human papillomavirus is a necessary cause of invasive cervical cancer worldwide. J Pathol 189: 12-19, 1999. 